\title{
Critérios diagnósticos e tratamento dos distúrbios respiratórios do sono: SAOS
}

\author{
Diagnostic criteria and treatment for \\ sleep-disordered breathing: obstructive sleep apnea syndrome \\ Lia Rita Azeredo Bittencourt, Eliazor Campos Caixeta
}

\begin{abstract}
Resumo
Neste capítulo são apresentadas as principais abordagens para o diagnóstico e tratamento dos pacientes com SAOS. 0 diagnóstico deve ser realizado através de uma minuciosa história clínica e exame físico. Para a confirmação diagnóstica, é necessária a realização da polissonografia completa de noite inteira sob supervisão, sendo que em pacientes cuja suspeita clínica é alta, registros simplificados domiciliares podem ser uma alternativa. 0 tratamento da SAOS requer medidas gerais e o uso de aparelhos de pressão positiva. Em casos moderados e graves, CPAP é o mais indicado, enquanto aparelhos intraorais são indicados em casos leves. Os procedimentos cirúrgicos são indicados quando alterações anatômicas são evidentes ou como auxílio aos demais tratamentos.
\end{abstract}

Descritores: Apneia do sono tipo obstrutiva/diagnóstico; Apneia do sono tipo obstrutiva/terapia; Apneia do sono tipo obstrutiva/cirurgia.

\begin{abstract}
In this chapter, the principal approaches to the diagnosis and treatment of patients with obstructive sleep apnea syndrome (OSAS) are presented. The diagnosis should be carried out by the taking of a thorough clinical history and by physical examination. For diagnostic confirmation, it is necessary to perform supervised overnight polysomnography. For patients in whom clinical suspicion is high, a simplified home study can be an alternative. The treatment of OSAS requires general measures and the use of positive pressure devices. In moderate and severe cases, CPAP is the method of choice, whereas oral appliances can be used in mild cases. Surgical procedures are recommended when anatomical alterations are evident or as an auxiliary method in combination with other types of treatment.
\end{abstract}

Keywords: Sleep apnea, obstructive/diagnosis; Sleep apnea, obstructive/treatment; Sleep apnea, obstructive/ surgery.

\section{Diagnóstico de SAOS}

0 diagnóstico da SAOS é baseado na história clínica, exame físico e testes de registro do sono (polissonografia e testes simplificados).

\section{Polissonografia de noite inteira}

0 estudo polissonográfico de noite inteira, realizado no laboratório sob supervisão de um técnico habilitado, constitui o método diagnóstico padrão para a avaliação dos distúrbios respiratórios do sono (nível de evidência 1; Figuras 1 e 2). ${ }^{(1)}$

Os principais critérios diagnósticos de SAOS se encontram no Quadro 1.(2)

\section{Polissonografia completa domiciliar}

É o registro polissonográfico completo, com ou sem supervisão de profissional habilitado, realizado no domicílio do paciente. Esse

exame é indicado para pacientes que não conseguem locomover-se para o laboratório do sono. Até o momento, não há evidências de que essa modalidade diagnóstica apresente vantagens sobre a polissonografia completa no laboratório.(3) Devido a complexidade do procedimento, risco de perda do exame, insegurança e desconforto do paciente e de seus familiares, julgamos que tal procedimento sempre deva ser realizado com supervisão.

\section{Monitoração cardiorrespiratória}

Esse tipo de monitorização é geralmente realizada no domicílio sem supervisão. Os parâmetros avaliados são FC, fluxo aéreo, oximetria e movimentos torácicos e abdominais. Recomenda-se que essa monitorização se restrinja a pacientes com forte suspeita de SAOS sem outras comorbidades ou outros distúrbios 


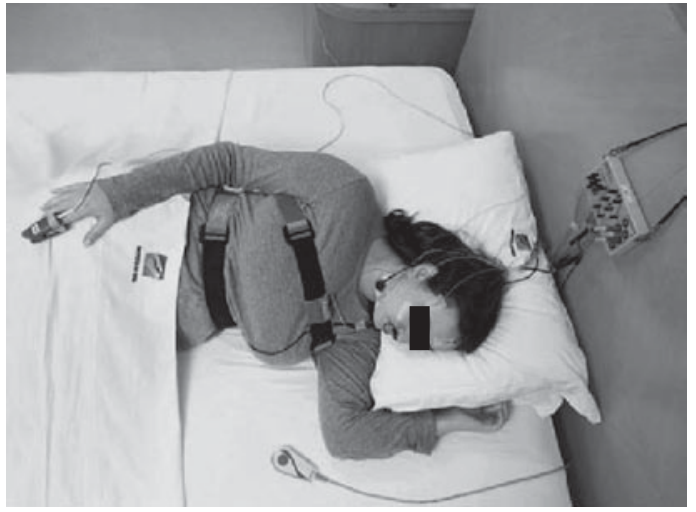

Figura 1 - Paciente realizando polissonografia.

do sono, quando a polissonografia padrão não é disponível ou quando o paciente não pode realizá-la no laboratório do sono devido a imobilidade, doença clínica ou por medida de segurança. Além disso, seu uso é recomendado no monitoramento do tratamento de SAOS que não o CPAP. Na eventualidade de exame negativo em pacientes com suspeita de SAOS, indica-se a polissonografia padrão no laboratório de sono. É importante salientar que a equipe deve estar ligada a um serviço de sono credenciado, que possibilitará a realização de uma polissonografia completa, se for necessário. ${ }^{(4)}$

\section{Polissonografia do tipo split-night}

A polissonografia do tipo split-night consiste, em uma mesma noite, de um registro inicial para o diagnóstico de SAOS, seguido de titulação da pressão positiva de via aérea. Esse exame é uma alternativa para casos graves de SAOS, e não se

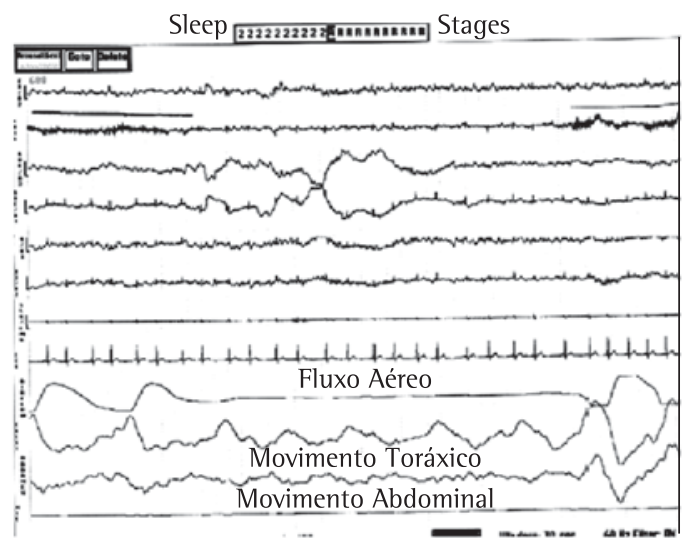

Figura 2 - Época de polissonografia demonstrando uma apneia obstrutiva. recomenda sua utilização de rotina. A grande limitação da polissonografia do tipo split-night é a possibilidade do ajuste incorreto do nível pressórico ideal, pois metade da noite pode ser insuficiente para titular adequadamente a pressão durante o sono REM e em posição supina. Além disso, considerando que o sono REM predomina na segunda metade da noite, os eventos respiratórios associados a ele podem não ser identificados adequadamente quando se faz o registro parcial. ${ }^{(1)}$

\section{Oximetria noturna}

Este é o registro isolado da oximetria de pulso, realizado com ou sem supervisão. A oximetria noturna apresenta baixa especificidade, não sendo recomendada para o diagnóstico de SAOS. ${ }^{(1)}$

\section{Polissonografia diurna (nap-study)}

Esse registro é realizado durante o dia, por um período de tempo geralmente curto. Seu resultado não é aceitável, pois subestima a presença e a gravidade de SAOS.(1)

\section{Tratamento de SAOS}

\section{Tratamento conservador por intermédio da higiene do sono e do emagrecimento}

Simples medidas, como a retirada de bebidas alcoólicas e de certas drogas (benzodiazepínicos, barbitúricos e narcóticos), a adequada posição do corpo e a perda de massa gorda, podem ser eficazes para o tratamento de SAOS. ${ }^{(5)}$ Também é importante evitar a posição do corpo na qual a apneia aparece ou piora (habitualmente o decúbito dorsal). ${ }^{(5)}$

\section{Tratamento farmacológico}

Alguns tratamentos farmacológicos, como a reposição hormonal nos indivíduos que apresentam acromegalia ou hipotireoidismo associados a SAOS, podem ser benéficos. (6-8) A reposição com hormônios femininos em mulheres na menopausa com SAOS tem mostrado um papel adicional para o tratamento dessa síndrome. ${ }^{(6)}$

0 tratamento farmacológico específico para SAOS, apesar dos vários estudos envolvendo diversos grupos farmacológicos, tem apresentado 
resultados controversos, não havendo ainda evidências clínicas sobre a sua efetividade. ${ }^{(6-8)}$

\section{CPAP}

Esses sistemas ainda permanecem como sendo a primeira escolha para o tratamento. 0 aparelho CPAP gera e direciona um fluxo contínuo de ar, (40-60 L/min), através de um tubo flexível, para uma máscara nasal ou nasobucal firmemente aderida à face do indivíduo. Quando a pressão positiva passa através das narinas, ocorre a dilatação de todo o trajeto das vias aéreas superiores (Figura 3).

Os benefícios do uso de CPAP estão relacionados à eliminação das apneias, ao aumento da saturação da oxi-hemoglobina e à diminuição dos despertares relacionados aos eventos respiratórios. Consequentemente, ocorre uma redução da sonolência diurna excessiva e melhora das funções neuropsíquicas, do desempenho subjetivo do trabalho, dos sintomas depressivos e da qualidade de vida. ${ }^{(9)} 0$ seu uso reduz as alterações cardiovasculares noturnas, podendo $^{(10)}$ ou não ${ }^{(11,12)}$ diminuir a hipertensão arterial diurna e a frequência de sofrer acidentes automobilísticos. Também melhora a sobrevida dos indivíduos com SAOS. Quando ajustado à pressão adequada, CPAP é quase sempre eficaz para o tratamento de SAOS. 0 fator que mais limita o seu uso é a sua não aceitação e adesão por parte do indivíduo. ${ }^{(9,10)}$

\section{Tratamento com aparelhos intraorais (AlOs)}

Existem atualmente dois modelos de AIOs utilizados para o controle de SAOS: os de avanço mandibular, que neste capítulo são denominados de AlOs (Figura 4), e os dispositivos de retenção lingual, os quais podem ser indicados para o tratamento do ronco primário por apresentarem uma menor adesão e eficácia do que os de avanço mandibular.

0 mecanismo de ação de um AlO se baseia na extensão/distensão das vias aéreas superiores pelo avanço da mandíbula. Essa distensão previne o colapso entre os tecidos da orofaringe e da base da língua, evitando o fechamento da via aérea superior, conforme se pode observar por meio de imagens e por videoendoscopia. ${ }^{(13)}$

Além de representar uma modalidade de tratamento não invasiva, ter um baixo custo,
Quadro 1 - Critérios diagnósticos de SAOS no adulto.

\begin{tabular}{|l|}
\hline \multicolumn{1}{|c|}{ Critérios (A + B + D) ou $(C+D)$ : } \\
diagnóstico de SAOS
\end{tabular}

ser reversível e de fácil confecção, os AlOs vêm sendo cada vez mais utilizados, com sucesso, para o tratamento do ronco primário, da resistência da via aérea superior e para o controle de SAOS leve. ${ }^{(2,14,15)}$ Esses aparelhos podem, comprovadamente, trazer melhora na qualidade de vida e do sono desses pacientes.

Antes do início do tratamento, o diagnóstico do distúrbio respiratório e a seleção dos pacientes favoráveis ao uso do aparelho devem ser conduzidos por um médico especialista em sono. É essencial também que o cirurgião

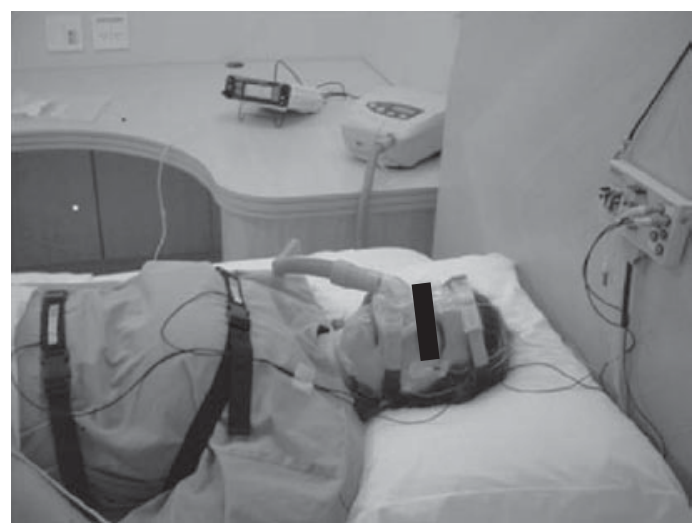

Figura 3 - Paciente realizando polissonografia para ajuste de pressão de CPAP. 


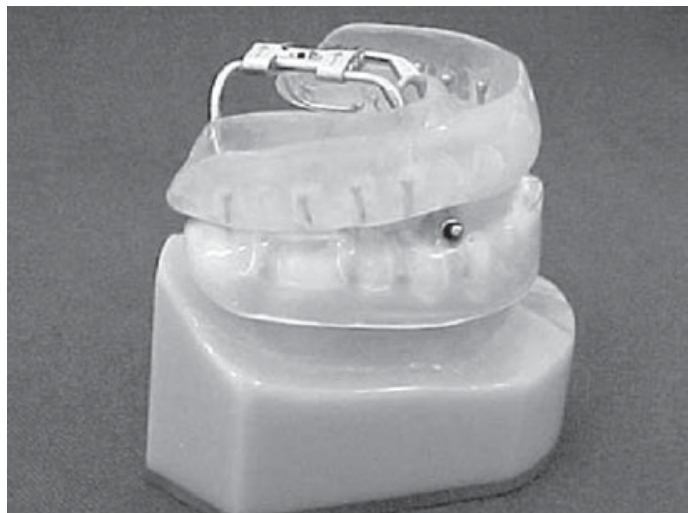

Figura 4 - Aparelho reposicionador mandibular.

dentista que irá conduzir o uso dos A10s tenha conhecimento sobre os possíveis efeitos colaterais a curto e longo prazo. Também podem ser uma opção de tratamento para os indivíduos com SAOS moderada e grave que não aceitam CPAP e para aqueles que são incapazes de tolerar ou que falharam nas tentativas do seu uso. ${ }^{(16)}$

\section{Tratamento cirúrgico}

As cirurgias direcionadas para SAOS têm por objetivo a modificação dos tecidos moles da faringe (palato, amígdalas, pilares amigdalianos e base da língua) e aqueles que abordam o esqueleto (maxila, mandíbula e hioide). Não existe um procedimento específico que possa resolver todas as necessidades do indivíduo e, muitas vezes, a combinação de cirurgias passa a ser a melhor forma de tratamento. Dependendo do problema anatômico a ser resolvido e da gravidade de SAOS, mais de uma modalidade cirúrgica pode ser utilizada de forma conjunta, num mesmo ato cirúrgico, ou de forma sequencial, na medida em que alguns benefícios são alcançados. A uvulopalatofaringoplastia, a primeira técnica cirúrgica proposta para 0 tratamento de SAOS, apresenta limitações porque trata somente da obstrução das vias aéreas superiores no nível retropalatal. Não são raras as complicações pós-cirúrgicas, como, entre outras, a insuficiência velofaríngea. ${ }^{(17)}$ Para evitar essas complicações, atualmente preconizam-se técnicas mais conservadoras, poupando a linha média do palato mole. Para o tratamento da macroglossia, a ressecção da parte posterior da língua por cirurgia aberta ou a laser foram tentativas de tratamento de SAOS. Entretanto, como não mostraram bons resultados, essas glossectomias não são usadas com frequência. ${ }^{(18)} A$ associação da técnica cirúrgica da uvulopalatofaringoplastia ao avanço do músculo genioglosso, assim como da miotomia do osso hioide, tem como objetivo dar uma maior tensão à língua, uma vez que não se cria espaço na cavidade faríngea, mas um posicionamento da língua sob tensão anteroposterior. Como os resultados são difíceis de prever, essa técnica deve ser utilizada com restrições. ${ }^{(19)}$ A cirurgia ortognática do avanço maxilomandibular proporciona uma anteriorização mais eficiente do palato e da base da língua. Com a utilização dessa técnica, 95-100\% dos indivíduos tiveram sucesso no tratamento da apneia obstrutiva do sono. ${ }^{(20)} \mathrm{Na}$ falha dos procedimentos cirúrgicos e do uso de CPAP, a traqueostomia pode ser necessária para os indivíduos com SAOS grave e complicações clínicas importantes. ${ }^{(21)} 0$ uso da radiofrequência para SAOS leve (palato e úvula) ou de moderado a severo (base da língua) pode trazer sucesso para o tratamento do ronco, ${ }^{(22)}$ embora a sua eficácia ainda não seja totalmente conhecida em relação ao tratamento de SAOS. Já as cirurgias nasais podem ser de grande auxílio para o uso de CPAP, mas nem tanto para o tratamento de SAOS. Os níveis pressóricos mais baixos de CPAP, após o tratamento cirúrgico nasal, têm mostrado resultados bastante animadores, principalmente para os casos em que as altas pressões dificultam ou até impedem o uso de CPAP nasal.(23)

Desse modo, a adequada seleção e avaliação dos pacientes é fundamental no sucesso do tratamento cirúrgico de SAOS.

\section{Referências}

1. Kushida CA, Littner MR, Morgenthaler T, Alessi CA, Bailey D, Coleman J Jr, et al. Practice parameters for the indications for polysomnography and related procedures: an update for 2005. Sleep. 2005;28(4):499-521.

2. American Academy of Sleep Medicine. The International Classification of Sleep Disorders: Diagnostic \& Coding Manual. Westchester: American Academy of Sleep Medicine; 2005.

3. Practice parameters for the use of portable recording in the assessment of obstructive sleep apnea. Standards of Practice Committee of the American Sleep Disorders Association. Sleep. 1994;17(4):372-7.

4. Collop NA, Anderson WM, Boehlecke B, Claman D, Goldberg R, Gottlieb DJ, et al. Clinical guidelines for the use of unattended portable monitors in the diagnosis of obstructive sleep apnea in adult patients. Portable Monitoring Task Force of the American Academy of Sleep Medicine. J Clin Sleep Med. 2007;3(7):737-47. 
5. Bittencourt LR, editor. Diagnóstico e tratamento da Síndrome da Apnéia Obstrutiva do Sono - Guia Prático. São Paulo: Livraria Médica Paulista; 2008.

6. Hachul H, Bittencourt LR, Andersen ML, Haidar MA, Baracat EC, Tufik S. Effects of hormone therapy with estrogen and/or progesterone on sleep pattern in postmenopausal women. Int J Gynaecol Obstet. 2008;103(3):207-12.

7. Abad VC, Guilleminault C. Pharmacological management of sleep apnoea. Expert Opin Pharmacother. 2006;7(1):11-23.

8. Smith 1, Lasserson TJ, Wright J. Drug therapy for obstructive sleep apnoea in adults. Cochrane Database Syst Rev. 2006;(2):CD003002.

9. Kushida CA, Littner MR, Hirshkowitz M, Morgenthaler Tl, Alessi CA, Bailey D, et al. Practice parameters for the use of continuous and bilevel positive airway pressure devices to treat adult patients with sleep-related breathing disorders. Sleep. 2006;29(3):375-80.

10. Pepperell JC, Ramdassingh-Dow S, Crosthwaite N, Mullins R, Jenkinson C, Stradling JR, et al. Ambulatory blood pressure after therapeutic and subtherapeutic nasal continuous positive airway pressure for obstructive sleep apnoea: a randomised parallel trial. Lancet. 2002;359(9302):204-10.

11. Barnes M, Houston D, Worsnop CJ, Neill AM, Mykytyn IJ, Kay A, et al. A randomized controlled trial of continuous positive airway pressure in mild obstructive sleep apnea. Am J Respir Crit Care Med. 2002;165(6):773-80.

12. Barnes M, McEvoy RD, Banks S, Tarquinio N, Murray CG, Vowles $\mathrm{N}$, et al. Efficacy of positive airway pressure and oral appliance in mild to moderate obstructive sleep apnea. Am J Respir Crit Care Med. 2004;170(6):656-64.

13. Hoffstein V. Review of oral appliances for treatment of sleep-disordered breathing. Sleep Breath. 2007;11(1):1-22.
14. Millman RP, Rosenberg CL, Carlisle CC, Kramer NR, Kahn DM, Bonitati AE. The efficacy of oral appliances in the treatment of persistent sleep apnea after uvulopalatopharyngoplasty. Chest. 1998;113(4):992-6.

15. Zonato A, Dal-Fabbro C, Bittencourt LR, Tufik S. A combined treatment for mild obstructive sleep apnea and hypopnea syndrome, a case report. Hypnos. 2001;2:24-8.

16. Martinho FL, Bittencourt LR, Gregório LC, Tufik S. Análise crítica da indicação do tratamento cirúrgico na SAOS. Pneumol Paulista. 2008;21:51

17. Altman JS, Halpert RD, Mickelson SA, Senior BA. Effect of uvulopalatopharyngoplasty and genial and hyoid advancement on swallowing in patients with obstructive sleep apnea syndrome. Otolaryngol Head Neck Surg. 1999;120(4):454-7.

18. Fujita S, Woodson BT, Clark JL, Wittig R. Laser midline glossectomy as a treatment for obstructive sleep apnea. Laryngoscope. 1991;101(8):805-9.

19. Riley RW, Powell NB, Guilleminault C. Obstructive sleep apnea and the hyoid: a revised surgical procedure. Otolaryngol Head Neck Surg. 1994;111(6):717-21.

20. Prinsell JR. Maxillomandibular advancement surgery in a site-specific treatment approach for obstructive sleep apnea in 50 consecutive patients. Chest. 1999;116(6):1519-29.

21. Partinen M, Jamieson A, Guilleminault C. Long-term outcome for obstructive sleep apnea syndrome patients. Mortality. Chest. 1988;94(6):1200-4.

22. Li KK, Powell NB, Riley RW, Troell RJ. Radiofrequency reduction of the palate: an extended follow up study. Otolaryngol Head Neck Surg 1999;121(2):P53-P54.

23. Zonato Al, Bittencourt LR, Martinho FL, Gregório LC, Tufik S. Upper airway surgery: the effect on nasal continuous positive airway pressure titration on obstructive sleep apnea patients. Eur Arch Otorhinolaryngol. 2006;263(5):481-6.

\section{Sobre os autores}

\section{Lia Rita Azeredo Bittencourt}

Professora Adjunta 111. Disciplina de Medicina e Biologia do Sono, Universidade Federal de São Paulo, São Paulo (SP) Brasil.

\section{Eliazor Campos Caixeta}

Professor de Clínica Médica 1. Disciplina de Pneumologia, Faculdade de Ciências Médicas de Minas Gerais. Belo Horizonte (MG) Brasil. E-mail para contato: lia@psicobio.epm.br (L. Bittencourt) ou eliazor@pulmonar.com.br (E. Caixeta) 